\section{Zur Fertilitätsprognose von Patienten mit testikulären und nichttestikulären Tumoren}

\author{
G. Schreiber, Uta-Christina Hipler, Sandra Däbritz \\ Universitätshautklinik Jena (Direktor: Prof. Dr. med. P. Elsner)
}

\begin{abstract}
Zusammenfassung. Moderne Therapieoptionen haben die Lebensprognose junger onkologischer Patienten verbessert, so dass Fragen der Familienplanung und des Kinderwunsches realistisch sind. Unsere Patienten mit testikulären (Gruppe 1) oder nichttestikulären (Gruppe 2) Tumorleiden weisen in Übereinstimmung mit der Literatur bereits vor der onkologischen Therapie in über $50 \%$ normabweichende Spermiogramme auf. Als Nebenwirkung der Onkotherapie von Hodentumoren ist klinisch vordergründig eine neurogene Spermatozoentransportstörung nach retroperitonealer Lymphadenektomie (RLA) zu nennen (über $30 \%$ ). Eine Chemotherapie wird in sehr unterschiedlicher Kombination, z.T. auch mit einer Radiatio eingesetzt und zeigt innerhalb des Beobachtungszeitraumes von 1 2 Jahren schwere Spermatogenesestörungen (50,3\% der Gruppe 1 resp. $100 \%$ der Gruppe 2). Eine mögliche Erholung der Spermatogenese nach ca. 2 Jahren lässt sich bei Hodentumorpatienten im Trend spermatologischer Befunde sowie einer FSH-Normalisierung erkennen. In der heterogenen Gruppe 2 kommt nur im Einzelfall eine Spermatogeneseerholung vor. Therapiestudien an größeren Patientengruppen sind erforderlich. Inhibin B und FSH weisen eine statistisch signifikante Korrelation auf, zur biologischen Signifikanz des Inhibin B als Prognosefaktor der Spermatogeneseerholung ist eine abschließende Beurteilung noch nicht möglich. Ausmaß und Reversibilität der Fertilitätsstörungen nach onkologischer Therapie sind bisher nicht sicher vorhersehbar, so dass wir in jedem Falle eine prätherapeutische Spermakryokonservierung empfehlen.
\end{abstract}

The Fertility Prognosis of Patients with Testicular and Non-Testicular Tumours. The prognosis of young oncological patients has been improved by modern therapeutic options, making issues such as family planning and having children more realistic. In accordance with the literature, $50 \%$ of our patients with testicular (group 1) and non-testicular (group 2) tumours show abnormal spermiograms prior to oncological therapy. A main side effect of oncotherapy of testicular tumours is a neurogenic disturbance of the transport of spermatozoa after RLA $(>30 \%)$. Chemotherapy is usually applied in various combinations, partially with radiation, and leads to severe disturbances of the spermatogenesis within $1-2$ years $(53,3 \%$ in group 1

Akt Dermatol 2001; 27: 269-272

(c) Georg Thieme Verlag Stuttgart · New York ISSN 0340-2541 and $100 \%$ in group 2). However, there is still a possible spermatogenesis recovery chance after 2 years, as indicated by spermatological evidence and normal FSH levels. This does not apply to patients of group 2 for whom recovery is rather an exception. Studies encompassing a larger number of patients are needed. Inhibin B and FSH show a statistically significant correlation. The role of Inhibin B as a prognostic factor of spermatogenesis is still discussed. Extent and reversibility of a disturbed fertility after oncological therapy are not predictable. Thus we strongly recommend a pre-therapeutic sperm cryopreservation.

\section{Einleitung}

Hodentumoren und maligne Lymphome sind die häufigsten onkologischen Erkrankungen des jüngeren Mannes mit jährlichen Inzidenzen um 6-8/100000 Männer in steigender Tendenz und Altersgipfeln im 3. Lebensjahrzehnt. Moderne Therapie-Optionen haben die Prognose quo ad vitam deutlich verbessert, so dass Fragen der Familienplanung und des Wunsches nach einem (weiteren) Kind realistisch sind. Allerdings ist die Fertilitätspotenz des onkologischen Patienten belastet: Vorbestehende und paraneoplastische Spermatogenesestörungen können sich kombinieren mit den Nebenwirkungen einer operativen und/oder aggressiven Chemo-Radiotherapie [1]. Verbindliche fertilitätsprognostische Faktoren sind bisher weitgehend unbekannt. Wir haben deshalb anlässlich der vorsorglichen Spermakryokonservierung und konsekutiver Kontrollen in jährlichen Abständen die spermatologischen Variablen, die Peptidhormone FSH und Inhibin B als Spermatogenesemarker [2,3] sowie das Gesamtserumtestosteron zur Beurteilung des Leydig-Zellkompartimentes bestimmt.

\section{Material und Methoden}

Erfasst wurden seit 199283 Patienten, die wegen onkologischer Therapien eine Fertilitätsvorsorge mit Hilfe der Spermakryokonservierung in Anspruch nahmen; Patienten mit bereits bestehender Azoospermie wurden nicht berücksichtigt. $51 \mathrm{~Pa}-$ tienten wiesen testikuläre Tumoren auf (Gruppe 1): $16 \times$ Tumoren mit mehr als einem histologischen Typ, $11 \times$ Seminom, $9 \times$ embryonales Karzinom, $4 \times$ Teratom, $11 \times($ noch $)$ nicht übermittelte Differenzierung. 32 Patienten hatten nichttestikuläre onkologische Erkrankungen (Gruppe 2): $14 \times$ malignes Hodgkin-Lymphom, $5 \times$ chronisch myeloische Leukämie (CML), 
$4 \times$ Non-Hodgkin-Lymphom, je $2 \times$ akute lymphatische Leukämie bzw. Ewing-Sarkom, je $1 \times$ Wilms-Tumor, Schilddrüsenkarzinom, malignes Pleuramesotheliom, Sigma- bzw. Analkarzinom. Alter, spermatologische Ausgangsbefunde sowie FSH, Inhibin B und Gesamtserumtestosteron bei Aufnahme sind in Tab. 1 zusammengestellt. Nach erfolgter Spermakryokonservierung wurden die Patienten in jährlichen Abständen zu Kontrolluntersuchungen einbestellt. Aus der Gruppe 1 war ein Patient innerhalb von 2 Jahren verstorben, aus der Gruppe 2 waren 4 Patienten mit CML im Zeitraum von einem halben bis 2 Jahren verstorben und sind in den Verlaufsbeobachtungen (Abb.1 -4) nicht enthalten. Zur Inhibin-B-Bestimmung wurde der Inhibin B-Dimer Assay Kit Serotec Oxford verwendet [4]. Die Korrelation zum FSH ergibt sich aus der Abb. 5.

\section{Ergebnisse}

Die spermatologischen Ausgangsbefunde sind bei Patienten der Gruppe 1 und 2 vergleichbar (Tab.1): Patienten mit testikulären Tumoren zeigen in 25/51 Fällen (49\%) eine Spermatozoenkonzentration < 20 Mio./ml, Patienten mit nichttestikulären Tumoren in 17/32 Fällen (53\%); p >0,05. Die Sertoli-Zellfunktion ist gekennzeichnet durch im Mittel höhere FSH-Werte in der Gruppe $1(\mathrm{p}<0,05)$ und relativ niedrigere Inhibin-BSpiegel. Da die Erfassung der Daten weiter zurückreicht als die initiale Inhibin-B-Bestimmung, liegen leider nicht für alle Patienten entsprechende Werte vor. Das Gesamtserumtestosteron ist in beiden Gruppen unauffällig.

Die onkologische Therapie beeinflusst offensichtlich den Verlauf der Befunde (Abb.1): 12/39 (31\%) Patienten der Gruppe 1 weisen eine Ejakulationsstörung auf, wenn im Anschluss an die Orchiektomie eine retroperitoneale Lymphadenektomie (RLA) erfolgte. Im Zeitraum bis zu 2 Jahren haben 16/30 $(53,3 \%)$ der spermatologisch auswertbaren Hodentumorpatienten insgesamt $<20$ Mio./ml Spermatozoen. Dabei zeigt erwartungsgemäß die Kombination Orchiektomie + RLA + Chemotherapie die gravierendsten Abweichungen. Im Beobachtungszeitraum von 2-6 Jahren haben bei einem leichten Erholungstrend 5/10 der spermatologisch befundeten Patienten $>20$ Mio./ml Spermatozoen. Alle mitgeteilten Ejakulationsstörungen der RLA-Patienten bestehen weiterhin.

In der Gruppe 2 (Abb. 2) führt Chemotherapie, z.T. kombiniert mit Radiotherapie im Nachbeobachtungszeitraum von ca. 1 Jahr in 17/20 (85\%) Fällen zur Azoospermie, dreimal zu einem schwergradigen OAT-Syndrom. Nach 2,3-6 Jahren zeigen 3/9 Patienten eine spermatologische Erholung, $4 \times$ besteht weiterhin Azoospermie.

Die medianen FSH-Werte der Gruppe 1 (Abb. 3) verändern sich über die Zeit hin nicht signifikant (Ausgangsbefund: 9,9; Nachbeobachtungszeit von 1-2 Jahren: 9,5; und 2-6 Jahre: $9,4 \mathrm{mIU} / \mathrm{ml}$ ), allerdings weisen sie in der Untergruppe Orchidektomie + RLA + Chemotherapie stärkere Auslenkungen auf $(12,0 \ldots 14,5 \ldots 10,2)$. Die Gruppe 2 (Abb. 4) zeigt dagegen ein deutliches medianes FSH-Inkrement von $14,6 \mathrm{mIU} / \mathrm{ml}$ innerhalb der ersten 2 Jahre und später einen geringen Rückgang auf $13,2 \mathrm{mIU} / \mathrm{ml}$.

Die Inhibin-B-Konzentration (Abb. 5) korreliert signifikant negativ $(\mathrm{p}<0,05)$ mit den FSH-Werten von Patienten der Gruppe 1 und 2 .

Tab.1 Ausgangswerte der Patienten mit testikulären (Gruppe 1) und nichttestikulären (Gruppe 2) Tumoren

\begin{tabular}{|c|c|c|c|c|c|c|c|}
\hline & $\mathrm{n}$ & $\begin{array}{l}\text { Alter } \\
\text { (n) }\end{array}$ & $\begin{array}{l}\text { Sp. Konz. } \\
\text { (Mio./ml) }\end{array}$ & $\begin{array}{l}\text { tot. Sp. Z. } \\
\text { (Mio.) }\end{array}$ & $\begin{array}{l}\text { FSH } \\
(\mathrm{mlU} / \mathrm{ml})\end{array}$ & $\begin{array}{l}\text { Inhibin B } \\
\text { (pg/ml) }\end{array}$ & $\begin{array}{l}\mathrm{T} \\
(\mathrm{nmol} / \mathrm{l})\end{array}$ \\
\hline Gruppe 1 & 51 & $27,3 \pm 5,3$ & $24,9 \pm 23,4$ & $88,2 \pm 101,3$ & $9,9 \pm 5,7$ & $131,9 \pm 38,8$ & $17,5 \pm 6,3$ \\
\hline \multirow[t]{2}{*}{ Gruppe 2} & 32 & $25,1 \pm 5,7$ & $34,2 \pm 41,6$ & $78,5 \pm 95,6$ & $4,8 \pm 2,5$ & $203,5 \pm 33,7$ & $14,6 \pm 5,9$ \\
\hline & & $<0,05$ & n.s. & n.s. & $<0,05$ & & n.s. \\
\hline
\end{tabular}

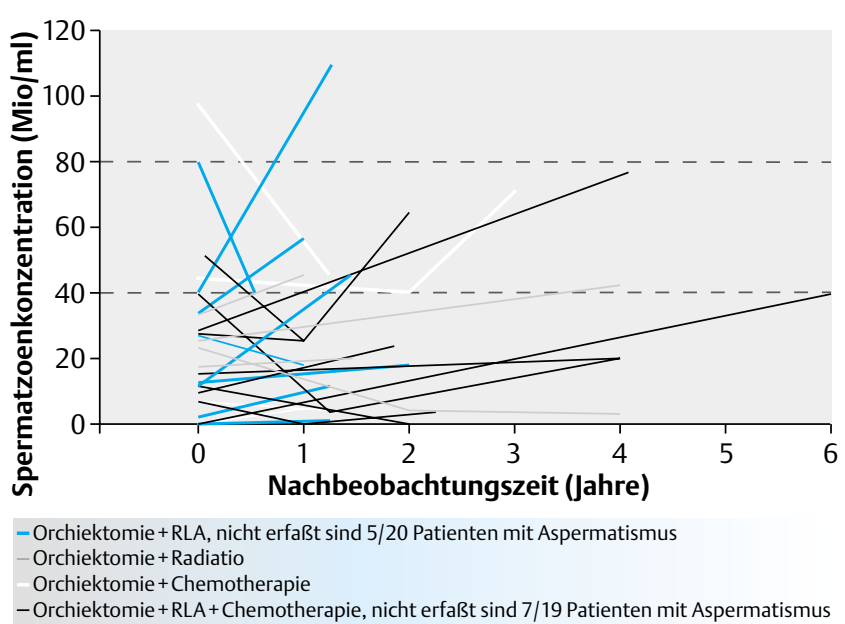

Abb.1 Spermatozoenkonzentration bei Patienten mit testikulären Tumoren.

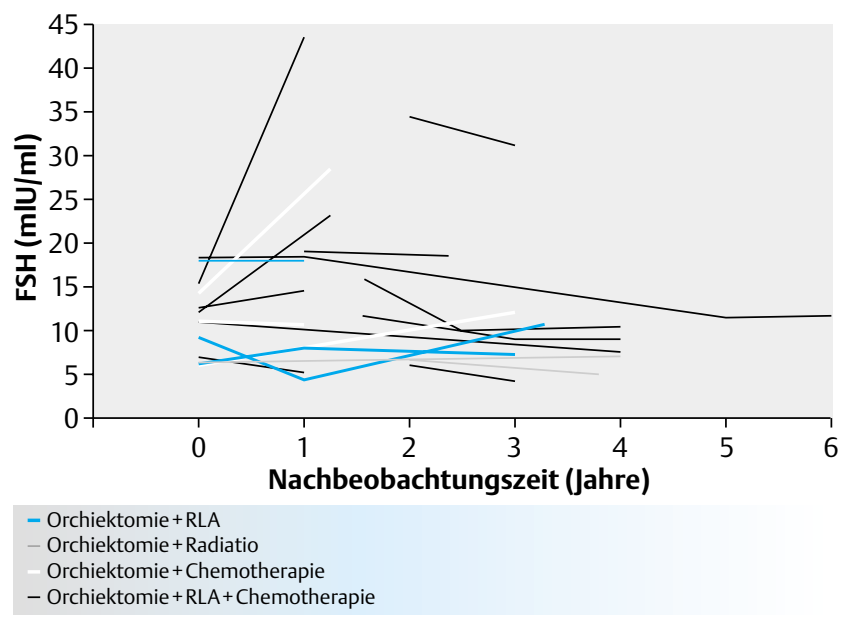

Abb. 2 FSH bei Patienten mit testikulären Tumoren. 


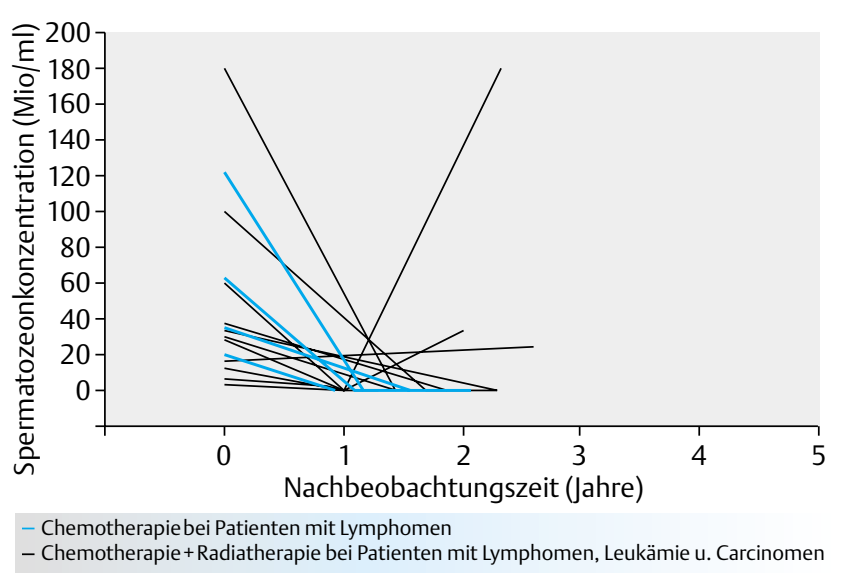

Abb. 3 Spermatozoenkonzentration bei Patienten mit nichttestikulären Tumoren.

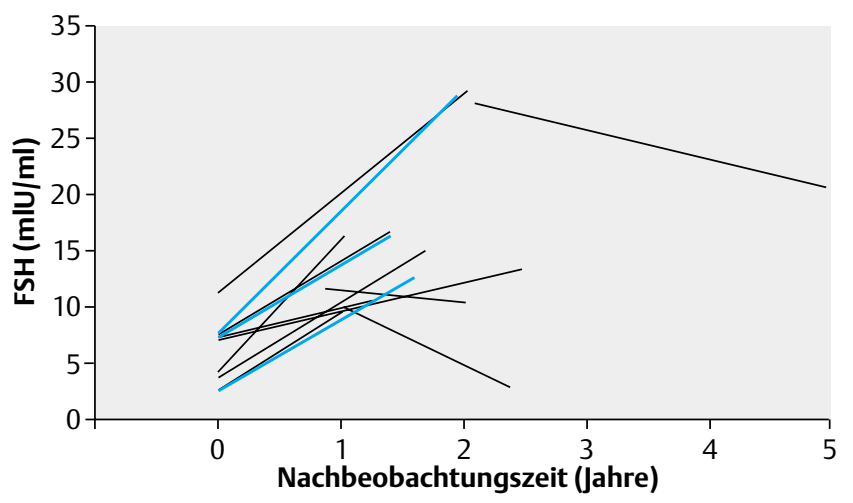

- Chemotherapie bei Patienten mit Lymphomen

- Chemotherapie+Radiatherapie bei Patienten mit Lymphomen, Leukämie u. Carcinomen

Abb. 4 FSH bei Patienten mit nichttestikulären Tumoren.

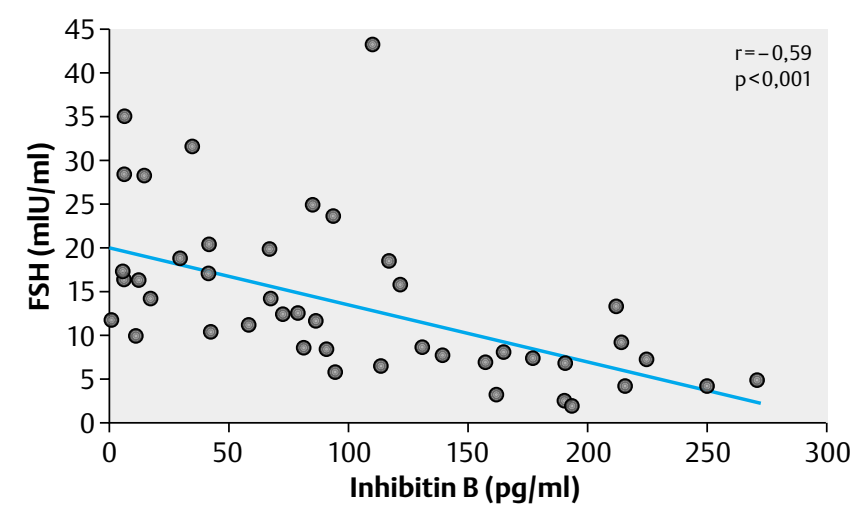

Abb. 5 Beziehung zwischen Inhibin B und FSH bei Patienten mit testikulären und nichttestikulären Tumoren.

\section{Diskussion}

Patienten mit Tumorleiden weisen sehr häufig bereits vor einer onkologischen Therapie normabweichende spermatologische Befunde auf. Oligozoospermien < 20 Mio./ml finden wir in 49 resp. $53 \%$ der testikulären bzw. nichttestikulären Tumorpatienten. Der Prozentsatz pathologischer Befunde erhöht sich zwangsläufig bei Berücksichtigung von Motilitätsstörungen und morphologischen Abweichungen sowie den Azoospermiefällen; letztere wurden hier nicht erfasst aus Gründen der anstehenden Spermakonservierung. Nach strenger Differenzierung finden Köhn und Schill [5] bei 137 Hodentumorpatienten in nur $18,2 \%$ eine Normozoospermie.

Die Ursache dafür ist weitgehend unklar. Zunächst ist an vorbestehende Störungen zu denken, wie sie auch im allgemeinen andrologischen Krankengut [6] zu finden und hier möglicherweise negativ selektioniert sind. Die mögliche Kombination mit paraneoplastischen Einflüssen (Fieber, Gewichtsverlust, Zytokine, Hormonaktivität) oder auch Nebeneffekten einer ausgedehnten Tumordiagnostik ist schwer zu differenzieren. Auffällig ist bei unseren Patienten ein signifikant erhöhter FSH-Wert in der Gruppe 1 gegenüber der Gruppe 2, interpretierbar als anlagebedingte oder onkogene tubuläre Insuffizienz des Hodentumorpatienten. Onkologische Therapieverfahren können die zum Ausgangspunkt erfasste Einschränkung der Fertilitätspotenz weiterhin gravierend verschlechtern. Inwiefern Erholungen dieser Kombinationsschäden möglich sind, ist bisher nicht sicher zu beantworten.

Operative Verfahren im Sinne der RLA wirken sich bei Durchtrennung sympathischer Nervenfasern aus Th12 bis L4 negativ auf den Ejakulationsvorgang aus. Nach radikaler RLA (Übersicht bei Hobisch et al. [7]) tritt der Verlust der antegraden Ejakulation in ca. $80 \%$ der Fälle ein. Unsere zugewiesenen Patienten mit Hodentumoren zeigen nach RLA in $31 \%$ eine Ejakulationsstörung, die über den bisherigen Nachbeobachtungszeitraum von 2 - 6 Jahren nicht reversibel war. Die Differenz deutet auf inzwischen übernommene Modifikationen der Operationstechnik hin. Eine weitere Verbesserung dieser Situation soll die konsequente RLA-Modifikation der „nerve sparing dissection“ mit 16\% Risiko einer Ejakulationsstörung [8] erreichen.

Eine Polychemotherapie führt in Abhängigkeit von den eingesetzten Substanzen und dem Dosis-Zeit-Regime zu ausgeprägten und oft irreversiblen Spermatogenesestörungen. Die Leydig-Zellen sind dagegen weitestgehend resistent gegenüber Zytostatika: Bei unseren Fällen lagen in diesem Sinne auch durchweg normale Serumtestosteronwerte vor. Die Spermiogrammabweichungen können bei unseren Patienten aufgrund der heterogenen Diagnoseverteilung und der sehr unterschiedlichen Therapieschemata, die z.T. auch eine Radiotherapie beinhalteten, nur vorsichtig interpretiert werden: Hodentumorpatienten zeigen in den Fällen mit erhaltener Ejakulationsfunktion nur eine geringe Zunahme der pathologischen Spermiogramme auf 53,3\% innerhalb von 2 Jahren, danach einen leichten Erholungstrend. Patienten mit nichttestikulären Tumorleiden, d.h. vorwiegend malignen Hodgkin- und NonHodgkin-Lymphomen, weisen dagegen bei insgesamt besseren Ausgangsbefunden eine schwere Spermatogenesedepression in $100 \%$ auf, nur im Einzelfall kommt es bei unseren Patienten nach 2-6 Jahren zur Erholung.

Die heute zur Behandlung der Hodentumoren eingesetzten Zytostatika (Cisplatin, Vinblastin, Etoposid, Bleomycin, Ifosfarnid) scheinen für das Keimepithel weniger toxisch zu sein. Pont et al. berichteten 1997 beispielsweise über spermatogeneseschonende Therapiezyklen mit einer kumulativen Dosis von $200 \mathrm{mg} / \mathrm{m}^{2}$ Cisplatin. Wegen erheblicher fertilitätsbelas- 
tender Nebenwirkungen des MOPP-Schemas (Mustargen, Vincristin, Procarbacin, Prednison) wird das maligne Hodgkin-Lymphom heute meist nach dem ABVD-Schema (Adriablastin, Bleomycin, Vinblastin, Dacarbacin) behandelt bzw. ein neues Schema, das BEACOPP-Schema (Bleomycin, Etoposid, Adriablastin, Cyclophosphamid, Procarbacin, Prednison) angewandt [9]. Zu Auswirkungen auf das Fertilitätspotenzial liegen dazu und zu den anderen von uns untersuchten wenigen Patienten der heterogenen Gruppe 2 bisher keine Studien vor.

Fertilitätsschädigende Einflüsse sind auch durch eine (zusätzliche) Radiotherapie zu kalkulieren. Ab $10 \mathrm{cGy}$ testikulärer Strahlendosis treten temporäre und ab 200 cGy irreversible Schäden auf. Nach Einschätzung von Albers [10] sollen zwei Drittel der Patienten nach prophylaktischer Radiatio eines Seminoms im Stadium I mit 26 Gy, das entspricht einer testikulären Streustrahlung bis zu 78 cGy (=1-3\% der Gesamtdosis) nach etwa 30 Monaten eine Spermatogenese-Restitution erreichen können.

Ob das Serum-FSH für Aussagen zur Fertilitätsprognose geeignet ist, lässt sich nicht sicher beantworten. Zunächst ist eine Erhöhung gebunden an die tubuläre Insuffizienz mit hochgradig gestörter oder fehlender Spermatogenese und gleichzeitiger hypophysärer Desinhibition, normales FSH signalisiert eine weitgehend normale Spermatogenese. Die diagnostische Sensitivität und Spezifität des FSH zum histologischen Erhaltungsgrad der Spermatogenese ist mit 88,9 bzw. 94,1\% sehr hoch [11]. Allerdings ist die Diskriminierung erhaltener gegenüber gestörter Spermatogenese damit nicht absolut verlässlich. Zum anderen fehlt ein Beleg darüber, dass eine Normalisierung des FSH als indirekter Hinweis auf die Sertoli-Zellfunktion auch mit einer Restitution der Spermatogenese korrespondiert, so dass die Suche nach anderen Markern sinnvoll erscheint. Das Inhibin B bietet gegenüber dem FSH den Vorteil des direkten Sertolizellproduktes [2,3,12,13]; es korreliert bei unseren Patienten signifikant mit dem FSH. Eine Absicherung bezüglich der prädiktiven Aussagen des Inhibin B ist erforderlich.

Eine Reihe methodischer Probleme (Therapiekombinationen, Ein- und Ausschlusskriterien, zahlreiche Confounder, standardisierte Spermadiagnostik, evaluierte Spermatogenesemarker, ausreichende Fallzahlen) erschweren eine fertilitätsprognostische Aussage, so dass nur im interdisziplinären, multizentrischen Zusammenschluss Ergebnisse erwartet werden können.

In Übereinstimmung mit anderen Autoren ist jedoch gegenwärtig für einen onkologischen Patienten mit geplanter aggressiver Polychemo/Radiotherapie eine sichere Einschätzung des Fertilitätspotenzials nicht möglich, so dass in jedem Fall eine Spermakryokonservierung zur Fertilitätsvorsorge anzuraten ist. Für den Fall der Spermatogeneseerholung kann die Konserve mit Einverständnis des Patienten verworfen werden. Im Falle irreversibler Schäden kann auf die Spermabank zur assistierten Fertilisierung zurückgegriffen werden, ohne den Patienten mit einer operativen Suche nach Spermatozoen im Nebenhoden (MESA) oder Hoden (TESE) zu belasten.

\section{Literatur}

${ }^{1}$ Petersen PM, Skakkebaek NE, Giwercman A. Gonadal function in men with testicular cancer: biological and clinical aspects. APMIS 1998; 106: 24-34

2 Anderson RA, Wallace EM, Groome NP, Bellis AJ, Wu FC. Physiological relationships between inhibin $B$, follicle stimulation hormon secretion and spermatogenesis in normal men and response to gonadotropin suppression by exogenous testosterone. Hum Reprod 1997; 12: 746-751

${ }^{3}$ Illingworth PJ, Groome NP, Byrd W, Rainey WE, McNeilly AS, Mather JP, Bremner WJ. Inhibin B: A likely candidate for the physiologically important form of inhibin in men. J Clin Endocrinol Metab 1996; 81: $1321-1325$

${ }^{4}$ Hipler U-C, Hochheim B, Knöll B, Tittelbach J, Schreiber G. Serum Inhibin B als Marker der Spermatogenese. Akt Dermatol 2001; 8/ 9: $273-278$

${ }^{5}$ Köhn FM, Schill WB. Kryospermabank München - Zwischenbilanz 1974-1986. Hautarzt 1998; 39: 91 -96

${ }^{6}$ Schreiber G. Aktuelle andrologische Aspekte der ungewollten Kinderlosigkeit. Akt Dermatol 1995; 21: 237-242

${ }^{7}$ Hobisch A, Janetschek G, Weißbach L. Tumoren des Hodens. In: Krause W, Weidner W (Hrsg). Andrologie. Stuttgart: Enke, 1998: $167-183$

${ }^{8}$ Jones DR, Norman AR, Harwich A, Hendry WF. Ejaculatory dysfunction after retroperitoneal lymphadenectomy. Eur Urol 1993; 23: $169-171$

${ }^{9}$ Diehl V. Morbus Hodgkin - neue Therapiestrategien. Target Forum 1998: 15 - 18

${ }^{10}$ Albers P. Fertilität nach Chemotherapie oder Radiatio. Fertilität 1998; 13 (Suppl 1): S24-S26

${ }^{11}$ Schreiber G, Görnig M, Zollmann C. Aktuelle Aspekte der Hormondiagnostik in der Andrologie - prädiktive Wertigkeiten zum Erhaltungsgrad der Spermatogenese. Wien Med Wochenschr 1997; 147: 84-89

12 Pierik FH, Vreeburg JTM, Stijnen T, de Jong FH, Weber RFA. Serum Inhibin B as a marker of spermatogenesis. J Clin Endocrinol Metab 1998; 83: 3110-3114

${ }^{13}$ Wallace EM, Groome NP, Riley SC, Parker AC, Wu FC. Effects of chemotherapy induced testicular damage on inhibin, gonadotropin, and testosterone secretion: a prospective longitudinal study. J Clin Endocrinol Metab 1997; 82: 3111 - 3115

14 Jockenhövel F. Hypogonadismus und Infertilität als Folge von allgemeinen Erkrankungen und Toxinen. Internist 1993; 34: 741 755

${ }^{15}$ Pont J, Albrecht W. Fertility after chemotherapy for testicular germ cell cancer. Fertil Steril 1997; 68: 1 -5

${ }^{16}$ Schwabe HR, Herrmann R, Mathew M, Graf KJ, Sander T, Cordes M, Nagel R, Weißbach L. The long-term toxicity of polychemotherapy in successfully treated testicular carcinoma. Dsch Med Wochenschr 1992; 117: $121-126$

\section{Prof. Dr. med. habil. Gerhard Schreiber}

Klinik für Dermatologie und Allergologie Abteilung Andrologie

Klinikum der Friedrich-Schiller-Universität

Erfurter Straße 35

07740 Jena 